University of New Mexico

UNM Digital Repository

2006

Unmatter Entities inside Nuclei, Predicted by the Brightsen

Nucleon Cluster Model

Florentin Smarandache

Dmitri Rabounski

Follow this and additional works at: https://digitalrepository.unm.edu/math_fsp

Part of the Atomic, Molecular and Optical Physics Commons 


\title{
Unmatter Entities inside Nuclei, Predicted by the Brightsen Nucleon Cluster Model
}

\author{
Florentin Smarandache and Dmitri Rabounski \\ Dept. of Mathematics and Science, Univ. of New Mexico, 200 College Road, Gallup, NM 87301, USA \\ E-mail: fsmarandache@yahoo.com; rabounski@yahoo.com
}

\begin{abstract}
Applying the R. A. Brightsen Nucleon Cluster Model of the atomic nucleus we discuss how unmatter entities (the conjugations of matter and antimatter) may be formed as clusters inside a nucleus. The model supports a hypothesis that antimatter nucleon clusters are present as a parton (sensu Feynman) superposition within the spatial confinement of the proton $\left({ }^{1} \mathrm{H}_{1}\right)$, the neutron, and the deuteron $\left({ }^{1} \mathrm{H}_{2}\right)$. If model predictions can be confirmed both mathematically and experimentally, a new physics is suggested. A proposed experiment is connected to othopositronium annihilation anomalies, which, being related to one of known unmatter entity, orthopositronium (built on electron and positron), opens a way to expand the Standard Model.
\end{abstract}

\section{Introduction}

According to Smarandache [1, 2, 3], following neutrosophy theory in philosophy and set theory in mathematics, the union of matter $<\mathrm{A}>$ and its antimatter opposite $<$ AntiA $>$ can form a neutral entity $<$ NeutA $>$ that is neither $<\mathrm{A}>$ nor $<$ AntiA $>$. The $<$ NeutA $>$ entity was termed "unmatter" by Smarandache [1] in order to highlight its intermediate physical constitution between matter and antimatter. Unmatter is formed when matter and antimatter baryons intermingle, regardless of the amount of time before the conjugation undergoes decay. Already Bohr long ago predicted the possibility of unmatter with his principle of complementarity, which holds that nature can be understood in terms of concepts that come in complementary pairs of opposites that are inextricably connected by a Heisenberg-like uncertainty principle. However, not all physical union of $\langle\mathrm{A}>$ with $<$ AntiA $>$ must form unmatter. For instance, the charge quantum number for the electron $\left(\mathrm{e}^{-}\right)$and its antimatter opposite positron $\left(\mathrm{e}^{+}\right)$make impossible the formation of a charge neutral state - the quantum situation must be either $\left(\mathrm{e}^{-}\right)$or $\left(\mathrm{e}^{+}\right)$.

Although the terminology "unmatter" is unconventional, unstable entities that contain a neutral union of matter and antimatter are well known experimentally for many years (e. g, pions, pentaquarks, positronium, etc.). Smarandache [3] presents numerous additional examples of unmatter that conform to formalism of quark quantum chromodynamics, already known since the 1970's. The basis that unmatter does exists comes from the 1970's experiments done at Brookhaven and CERN [4-8], where unstable unmatter-like entities were found. Recently "physicists suspect they have created the first molecules from atoms that meld matter with antimatter. Allen Mills of the University of California, Riverside, and his colleagues say they have seen telltale signs of positronium molecules, made from two positronium atoms" $[9,10]$. A bound and quasi-stable unmatter baryon- ium has been verified experimentally as a weak resonance between a proton and antiproton using a Skyrme-type model potential. Further evidence that neutral entities derive from union of opposites comes from the spin induced magnetic moment of atoms, which can exist in a quantum state of both spin up and spin down at the same time, a quantum condition that follows the superposition principal of physics. In quantum physics, virtual and physical states that are mutually exclusive while simultaneously entangled, can form a unity of opposites $<$ NeutA $>$ via the principle of superposition.

Our motivation for this communication is to the question: would the superposition principal hold when mass symmetrical and asymmetrical matter and antimatter nucleon wavefunctions become entangled, thus allowing for possible formation of macroscopic "unmatter" nucleon entities, either stable or unstable? Here we introduce how the novel Nucleon Cluster Model of the late R. A. Brightsen [11-17] does predict formation of unmatter as the product of such a superposition between matter and antimatter nucleon clusters. The model suggests a radical hypothesis that antimatter nucleon clusters are present as a hidden parton type variable (sensu Feynman) superposed within the spatial confinement of the proton $\left({ }^{1} \mathrm{H}_{1}\right)$, the neutron, and the deuteron $\left({ }^{1} \mathrm{H}_{2}\right)$. Because the mathematics involving interactions between matter and antimatter nucleon clusters is not developed, theoretical work will be needed to test model predictions. If model predictions can be experimentally confirmed, a new physics is suggested.

\section{The Brightsen Nucleon Cluster Model to unmatter entities inside nuclei}

Of fundamental importance to the study of nuclear physics is the attempt to explain the macroscopic structural phenomena of the atomic nucleus. Classically, nuclear structure mathematically derives from two opposing views: (1) that the proton $[\mathrm{P}]$ and neutron $[\mathrm{N}]$ are independent (unbound) interacting 


\begin{tabular}{|c|c|c|c|c|c|c|c|}
\hline $\begin{array}{l}\text { Matter } \\
\text { Clusters } \longrightarrow \\
\text { Antimatter } \\
\text { Clusters } \downarrow\end{array}$ & $\begin{array}{c}{[\mathrm{NP}]} \\
\text { Deuteron } \\
\mathrm{i} \\
\text { Stable }\end{array}$ & $\begin{array}{c}{[\mathrm{NPN}]} \\
\text { Triton } \\
\mathrm{j} \\
\text { Beta-unstable }\end{array}$ & $\begin{array}{c}{[\mathrm{PNP}]} \\
\text { Helium-3 } \\
\text { k } \\
\text { Stable }\end{array}$ & $\begin{array}{c}{[\mathrm{NN}]} \\
\text { Di-Neutron } \\
1\end{array}$ & $\begin{array}{c}{[\mathrm{PP}]} \\
\text { Di-Proton } \\
\mathrm{m}\end{array}$ & $\begin{array}{c}{[\mathrm{NNN}]} \\
\text { Tri-Neutron } \\
\mathrm{n}\end{array}$ & $\begin{array}{c}{[\mathrm{PPP}]} \\
\text { Tri-Proton } \\
\quad \mathrm{o}\end{array}$ \\
\hline $\begin{array}{c}{\left[\mathrm{N}^{\wedge} \mathrm{P}^{\wedge}\right]} \\
\text { a } \\
\text { Stable }\end{array}$ & & $\begin{array}{c}{[\mathrm{N}]} \\
|\mathrm{NP}|\left|\mathrm{N}^{\wedge} \mathrm{P}^{\wedge}\right|\end{array}$ & $\begin{array}{c}{[\mathrm{P}]} \\
|\mathrm{NP}|\left|\mathrm{N}^{\wedge} \mathrm{P}^{\wedge}\right|\end{array}$ & $\begin{array}{l}\text { Pions } \\
\left(\mathrm{qq}^{\wedge}\right)\end{array}$ & $\begin{array}{l}\text { Pions } \\
\left(\mathrm{q} \mathrm{q}^{\wedge}\right)\end{array}$ & $\begin{array}{c}{[\mathrm{N}]} \\
|\mathrm{NN}|\left|\mathrm{N}^{\wedge} \mathrm{P}^{\wedge}\right|\end{array}$ & $\begin{array}{c}{[\mathrm{P}]} \\
\left|\mathrm{N}^{\wedge} \mathrm{P}^{\wedge}\right||\mathrm{PP}|\end{array}$ \\
\hline $\begin{array}{c}{\left[\mathrm{N}^{\wedge} \mathrm{P}^{\wedge} \mathrm{N}^{\wedge}\right]} \\
b \\
\text { Beta-unstable }\end{array}$ & $\begin{array}{c}{\left[\mathrm{N}^{\wedge}\right]} \\
|\mathrm{NP}|\left|\mathrm{N}^{\wedge} \mathrm{P}^{\wedge}\right|\end{array}$ & & $\begin{array}{l}\text { Pions } \\
\left(\mathrm{qq}^{\wedge}\right)\end{array}$ & $\begin{array}{c}{\left[\mathrm{P}^{\wedge}\right]} \\
|\mathrm{NN}|\left|\mathrm{N}^{\wedge} \mathrm{N}^{\wedge}\right|\end{array}$ & $\begin{array}{c}{\left[\mathrm{N}^{\wedge}\right]} \\
\left|\mathrm{N}^{\wedge} \mathrm{P}^{\wedge}\right||\mathrm{PP}|\end{array}$ & $\begin{array}{l}\text { Pions } \\
\left(\mathrm{qq}^{\wedge}\right)\end{array}$ & $\begin{array}{c}\text { Tetraquarks } \\
\left(\mathrm{qqq}^{\wedge} \mathrm{q}^{\wedge}\right)\end{array}$ \\
\hline $\begin{array}{c}{\left[\mathrm{P}^{\wedge} \mathrm{N}^{\wedge} \mathrm{P}^{\wedge}\right]} \\
\mathrm{c} \\
\text { Stable }\end{array}$ & $\begin{array}{c}{\left[\mathrm{P}^{\wedge}\right]} \\
|\mathrm{NP}|\left|\mathrm{N}^{\wedge} \mathrm{P}^{\wedge}\right|\end{array}$ & $\begin{array}{l}\text { Pions } \\
\left(\mathrm{qq}^{\wedge}\right)\end{array}$ & & $\begin{array}{c}{\left[\mathrm{P}^{\wedge}\right]} \\
\left|\mathrm{N}^{\wedge} \mathrm{P}^{\wedge}\right||\mathrm{NN}|\end{array}$ & $\begin{array}{c}{\left[\mathrm{N}^{\wedge}\right]} \\
|\mathrm{PP}|\left|\mathrm{P}^{\wedge} \mathrm{P}^{\wedge}\right|\end{array}$ & 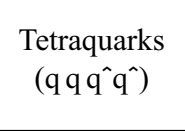 & $\begin{array}{l}\text { Pions } \\
\left(q q^{\wedge}\right)\end{array}$ \\
\hline $\begin{array}{c}{\left[\mathrm{N}^{\wedge} \mathrm{N}^{\wedge}\right]} \\
\mathrm{d}\end{array}$ & $\begin{array}{l}\text { Pions } \\
\left(\mathrm{q} \mathrm{q}^{\wedge}\right)\end{array}$ & $\begin{array}{c}{[\mathrm{N}]} \\
|\mathrm{NN}|\left|\mathrm{N}^{\wedge} \mathrm{N}^{\wedge}\right|\end{array}$ & $\begin{array}{c}{[\mathrm{P}]} \\
|\mathrm{NP}| \mathrm{N}^{\wedge} \mathrm{N}^{\wedge} \mid\end{array}$ & & $\begin{array}{l}\text { Tetraquarks } \\
\left(\mathrm{qqq}^{\wedge} \mathrm{q}^{\wedge}\right)\end{array}$ & $\begin{array}{c}{[\mathrm{N}]} \\
|\mathrm{NN}|\left|\mathrm{N}^{\wedge} \mathrm{N}^{\wedge}\right|\end{array}$ & $\begin{array}{c}{[\mathrm{P}]} \\
|\mathrm{PP}|\left|\mathrm{N}^{\wedge} \mathrm{N}^{\wedge}\right|\end{array}$ \\
\hline $\begin{array}{c}{\left[\mathrm{P}^{\wedge} \mathrm{P}^{\wedge}\right]} \\
\mathrm{e}\end{array}$ & $\begin{array}{l}\text { Pions } \\
\left(\mathrm{qq} \mathrm{q}^{\wedge}\right)\end{array}$ & $\begin{array}{c}{[\mathrm{N}]} \\
|\mathrm{NP}|\left|\mathrm{P}^{\wedge} \mathrm{P}^{\wedge}\right|\end{array}$ & $\begin{array}{c}{[\mathrm{P}]} \\
|\mathrm{NP}|\left|\mathrm{P}^{\wedge} \mathrm{P}^{\wedge}\right|\end{array}$ & $\begin{array}{l}\text { Tetraquarks } \\
\left(\mathrm{qq}^{\wedge} \mathrm{q}^{\wedge}\right)\end{array}$ & & $\begin{array}{c}{[\mathrm{N}]} \\
\left|\mathrm{P}^{\wedge} \mathrm{P}^{\wedge}\right||\mathrm{NN}|\end{array}$ & $\begin{array}{c}{[\mathrm{P}]} \\
|\mathrm{PP}|\left|\mathrm{P}^{\wedge} \mathrm{P}^{\wedge}\right|\end{array}$ \\
\hline $\begin{array}{c}{\left[\mathrm{N}^{\wedge} \mathrm{N}^{\wedge} \mathrm{N}^{\wedge}\right]} \\
\mathrm{f}\end{array}$ & $\begin{array}{c}{\left[\mathrm{N}^{\wedge}\right]} \\
|\mathrm{NP}|\left|\mathrm{N}^{\wedge} \mathrm{N}^{\wedge}\right|\end{array}$ & $\begin{array}{l}\text { Pions } \\
\left(\mathrm{qq}^{\wedge}\right)\end{array}$ & $\begin{array}{l}\text { Tetraquarks } \\
\left(\mathrm{qqq}^{\wedge} \mathrm{q}^{\wedge}\right)\end{array}$ & $\begin{array}{c}{\left[\mathrm{N}^{\wedge}\right]} \\
|\mathrm{NN}|\left|\mathrm{N}^{\wedge} \mathrm{N}^{\wedge}\right|\end{array}$ & $\begin{array}{c}{\left[\mathrm{N}^{\wedge}\right]} \\
\left|\mathrm{N}^{\wedge} \mathrm{N}^{\wedge}\right| \\
|\mathrm{PP}|\end{array}$ & & 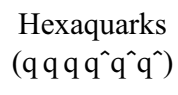 \\
\hline $\begin{array}{c}{\left[\mathrm{P}^{\wedge} \mathrm{P}^{\wedge} \mathrm{P}^{\wedge}\right]} \\
\mathrm{g}\end{array}$ & $\begin{array}{c}{\left[\mathrm{P}^{\wedge}\right]} \\
|\mathrm{NP}|\left|\mathrm{P}^{\wedge} \mathrm{P}^{\wedge}\right|\end{array}$ & $\begin{array}{l}\text { Tetraquarks } \\
\left(\mathrm{qqq}^{\wedge} \mathrm{q}^{\wedge}\right)\end{array}$ & $\begin{array}{l}\text { Pions } \\
\left(\mathrm{q}^{\wedge}\right)\end{array}$ & $\begin{array}{c}{\left[\mathrm{P}^{\wedge}\right]} \\
\left|\mathrm{P}^{\wedge} \mathrm{P}^{\wedge}\right||\mathrm{NN}|\end{array}$ & $\begin{array}{c}{\left[\mathrm{P}^{\wedge}\right]} \\
\left|\mathrm{P}^{\wedge} \mathrm{P}^{\wedge}\right||\mathrm{PP}|\end{array}$ & 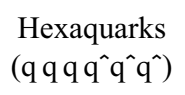 & \\
\hline
\end{tabular}

Table 1: Unmatter entities (stable, quasi-stable, unstable) created from union of matter and antimatter nucleon clusters as predicted by the gravity-antigravity formalism of the Brightsen Nucleon Cluster Model. Shaded cells represent interactions that result in annihilation of mirror opposite two- and three- body clusters. Top nucleons within cells show superposed state comprised of three valance quarks; bottom structures show superposed state of hidden unmatter in the form of nucleon clusters. Unstable pions, tetraquarks, and hexaquark unmatter are predicted from union of mass symmetrical clusters that are not mirror opposites. The symbol ${ }^{\wedge}=$ antimatter, $\mathrm{N}=$ neutron, $\mathrm{P}$ = proton, $\mathrm{q}=$ quark. (Communication with R. D. Davic).

fermions within nuclear shells, or (2) that nucleons interact collectively in the form of a liquid-drop. Compromise models attempt to cluster nucleons into interacting [NP] boson pairs (e.g., Interacting Boson Model-IBM), or, as in the case of the Interacting Boson-Fermion Model (IBFM), link boson clusters [NP] with un-paired and independent nucleons [P] and $[\mathrm{N}]$ acting as fermions.

However, an alternative view, at least since the 1937 Resonating Group Method of Wheeler, and the 1965 ClosePacked Spheron Model of Pauling, holds that the macroscopic structure of atomic nuclei is best described as being composed of a small number of interacting boson-fermion nucleon "clusters" (e. g., helium-3 [PNP], triton [NPN], deuteron $[\mathrm{NP}])$, as opposed to independent $[\mathrm{N}]$ and $[\mathrm{P}]$ nucleons acting as fermions, either independently or collectively. Mathematically, such clusters represent a spatially localized mass-charge-spin subsystem composed of strongly correlated nucleons, for which realistic two- and three body wave functions can be written. In this view, quark-gluon dynamics are confined within the formalism of 6-quark bags [NP] and 9-quark bags ([PNP] and [NPN]), as opposed to valance quarks forming free nucleons. The experimental evidence in support of nucleons interacting as boson-fermion clusters is now extensive and well reviewed.

One novel nucleon cluster model is that of R. A. Brightsen, which was derived from the identification of masscharge symmetry systems of isotopes along the Z-N Serge plot. According to Brightsen, all beta-stable matter and antimatter isotopes are formed by potential combinations of two- and three nucleon clusters; e.g., ([NP], [PNP], [NPN], $[\mathrm{NN}],[\mathrm{PP}],[\mathrm{NNN}],[\mathrm{PPP}]$, and/or their mirror antimatter clusters $\left[\mathrm{N}^{\wedge} \mathrm{P}^{\wedge}\right],\left[\mathrm{P}^{\wedge} \mathrm{N}^{\wedge} \mathrm{P}^{\wedge}\right],\left[\mathrm{N}^{\wedge} \mathrm{P}^{\wedge} \mathrm{N}^{\wedge}\right],\left[\mathrm{N}^{\wedge} \mathrm{N}^{\wedge}\right],\left[\mathrm{P}^{\wedge} \mathrm{P}^{\wedge}\right],\left[\mathrm{P}^{\wedge} \mathrm{P}^{\wedge} \mathrm{P}^{\wedge}\right]$, $\left[\mathrm{N}^{\wedge} \mathrm{N}^{\wedge} \mathrm{N}^{\wedge}\right]$, where the symbol ${ }^{\wedge}$ here is used to denote antimatter. A unique prediction of the Brightsen model is that a stable union must result between interaction of mass asymmetrical matter (positive mass) and antimatter (negative mass) nucleon clusters to form protons and neutrons, for example the interaction between matter [PNP] + antimatter 
$\left[\mathrm{N}^{\wedge} \mathrm{P}^{\wedge}\right]$. Why union and not annihilation of mass asymmetrical matter and antimatter entities? As explained by Brightsen, independent (unbound) neutron and protons do not exist in nuclear shells, and the nature of the mathematical series of cluster interactions $(3[\mathrm{NP}]$ clusters $=1[\mathrm{NPN}]$ cluster +1 [PNP] cluster), makes it impossible for matter and antimatter clusters of identical mass to coexist in stable isotopes. Thus, annihilation cannot take place between mass asymmetrical two- and three matter and antimatter nucleon clusters, only strong bonding (attraction).

Here is the Table that tells how unmatter may be formed from nucleon clusters according to the Brightsen model.

\section{A proposed experimental test}

As known, Standard Model of Quantum Electrodynamics explains all known phenomena with high precision, aside for anomalies in orthopositronium annihilation, discovered in 1987.

The Brightsen model, like many other models (see References), is outside the Standard Model. They all pretend to expand the Standard Model in one or another way. Therefore today, in order to judge the alternative models as true or false, we should compare their predictions to orthopositronium annihilation anomalies, the solely unexplained by the Standard Model. Of those models the Brightsen model has a chance to be tested in such way, because it includes unmatter entities (the conjugations of particles and anti-particles) inside an atomic nucleus that could produce effect in the forming of orthopositronium by $\beta^{+}$-decay positrons and its annihilation.

In brief, the anomalies in orthopositronium annihilation are as follows.

Positronium is an atom-like orbital system that includes an electron and its anti-particle, positron, coupled by electrostatic forces. There are two kinds of that: parapositronium ${ }^{\mathrm{S}} \mathrm{Ps}$, in which the spins of electron and positron are oppositely directed and the summary spin is zero, and orthopositronium ${ }^{\mathrm{T}} \mathrm{Ps}$, in which the spins are co-directed and the summary spin is one. Because a particle-antiparticle (unmatter) system is unstable, life span of positronium is rather small. In vacuum, parapositronium decays in $\tau \simeq 1.25 \times 10^{-10} \mathrm{~s}$, while orthopositronium is $\tau \simeq 1.4 \times 10^{-7} \mathrm{~s}$ after the birth. In a medium the life span is even shorter because positronium tends to annihilate with electrons of the media.

In laboratory environment positronium can be obtained by placing a source of free positrons into a matter, for instance, one-atom gas. The source of positrons is $\beta^{+}$-decay, self-triggered decays of protons in neutron-deficient atoms*

$$
\mathrm{p} \rightarrow \mathrm{n}+\mathrm{e}^{+}+\nu_{\mathrm{e}}
$$

Some of free positrons released from $\beta^{+}$-decay source

${ }^{*}$ It is also known as positron $\beta^{+}$-decay. During $\beta^{-}$-decay in nucleus neutron decays $\mathrm{n} \rightarrow \mathrm{p}+\mathrm{e}^{-}+\tilde{\nu}_{\mathrm{e}}$. into gas quite soon annihilate with free electrons and electrons in the container's walls. Other positrons capture electrons from gas atoms thus producing orthopositronium and parapositronium (in 3:1 statistical ratio). Time spectrum of positrons (number of positrons vs. life span) is the basic characteristic of their annihilation in matter.

In inert gases the time spectrum of annihilation of free positrons generally reminds of exponential curve with a plateau in its central part, known as "shoulder" [29, 30]. In 1965 Osmon published [29] pictures of observed time spectra of annihilation of positrons in inert gases ( $\mathrm{He}, \mathrm{Ne}, \mathrm{Ar}, \mathrm{Kr}$, $\mathrm{Xe}$ ). In his experiments he used ${ }^{22} \mathrm{NaCl}$ as a source of $\beta^{+}$. decay positrons. Analyzing the results of the experiments, Levin noted that the spectrum in neon was peculiar compared to those in other one-atom gases: in neon points in the curve were so widely scattered, that presence of a "shoulder" was unsure. Repeated measurements of temporal spectra of annihilation of positrons in $\mathrm{He}, \mathrm{Ne}$, and $\mathrm{Ar}$, later accomplished by Levin [31,32], have proven existence of anomaly in neon. Specific feature of the experiments done by Osmon, Levin and some other researchers in the UK, Canada, and Japan is that the source of positrons was ${ }^{22} \mathrm{Na}$, while the moment of birth of positron was registered according to $\gamma_{n}$ quantum of decay of excited ${ }^{22 *} \mathrm{Ne}$

$$
{ }^{22 *} \mathrm{Ne} \rightarrow{ }^{22} \mathrm{Ne}+\gamma_{\mathrm{n}}
$$

from one of products of $\beta^{+}$-decay of ${ }^{22 *} \mathrm{Na}$.

In his experiments $[33,34]$ Levin discovered that the peculiarity of annihilation spectrum in neon (abnormally wide scattered points) is linked to presence in natural neon of substantial quantity of its isotope ${ }^{22} \mathrm{Ne}$ (around 9\%). Levin called this effect isotope anomaly. Temporal spectra were measured in neon environments of two isotopic compositions: (1) natural neon $\left(90.88 \%\right.$ of ${ }^{20} \mathrm{Ne}, 0.26 \%$ of ${ }^{21} \mathrm{Ne}$, and $8.86 \%$ of ${ }^{22} \mathrm{Ne}$ ); (2) neon with reduced content of ${ }^{22} \mathrm{Ne}$ $\left(94.83 \%\right.$ of ${ }^{20} \mathrm{Ne}, 0.22 \%$ of ${ }^{21} \mathrm{Ne}$, and $4.91 \%$ of $\left.{ }^{22} \mathrm{Ne}\right)$. Comparison of temporal spectra of positron decay revealed: in natural neon (the 1st composition) the shoulder is fuzzy, while in neon poor with ${ }^{22} \mathrm{Ne}$ (the 2 nd composition) the shoulder is always clearly pronounced. In the part of spectrum, to which ${ }^{\mathrm{T}}$ Ps-decay mostly contributes, the ratio between intensity of decay in poor neon and that in natural neon (with much isotope ${ }^{22} \mathrm{Ne}$ ) is $1.85 \pm 0.1$ [34].

Another anomaly is substantially higher measured rate of annihilation of orthopositronium (the value reciprocal to its life span) compared to that predicted by QED.

Measurement of orthopositronium annihilation rate is among the main tests aimed to experimental verification of QED laws of conservation. In 1987 thanks to new precision technology a group of researchers based in the University of Michigan (Ann Arbor) made a breakthrough in this area. The obtained results showed substantial gap between experiment and theory. The anomaly that the Michigan group revealed 
was that measured rates of annihilation at $\lambda_{\mathrm{T}(\exp )}=7.0514 \pm$ $\pm 0.0014 \mu \mathrm{s}^{-1}$ and $\lambda_{\mathrm{T}(\exp )}=7.0482 \pm 0.0016 \mu \mathrm{s}^{-1}$ (with unseen-before precision of $0.02 \%$ and $0.023 \%$ using vacuum and gas methods [35-38]) were much higher compared to $\lambda_{\mathrm{T} \text { (theor) }}=7.00383 \pm 0.00005 \mu \mathrm{s}^{-1}$ as predicted by QED [39-42]. The effect was later called $\lambda_{\mathrm{T}}$-anomaly [43].

Theorists foresaw possible annihilation rate anomaly not long before the first experiments were accomplished in Michigan. In 1986 Holdom [44] suggested that "mixed type" particles may exist, which being in the state of oscillation stay for some time in our world and for some time in the mirror Universe, possessing negative masses and energies. In the same year Glashow [45] gave further development to the idea and showed that in case of 3-photon annihilation ${ }^{\mathrm{T}} \mathrm{Ps}$ will "mix up" with its mirror twin thus producing two effects: (1) higher annihilation rate due to additional mode of decay ${ }^{\mathrm{T}} \mathrm{Ps} \rightarrow$ nothing, because products of decay passed into the mirror Universe can not be detected; (2) the ratio between orthopositronium and parapositronium numbers will decrease from ${ }^{\mathrm{T}} \mathrm{Ps}_{\mathrm{s}}{ }^{\mathrm{S}} \mathrm{Ps}=3: 1$ to $1.5: 1$. But at that time (in 1986) Glashow concluded that no interaction is possible between our-world and mirror-world particles.

On the other hand, by the early 1990's these theoretic studies encouraged many researchers worldwide for experimental search of various "exotic" (unexplained in QED) modes of TPs-decay, which could lit some light on abnormally high rate of decay. These were, to name just a few, search for ${ }^{\mathrm{T}} \mathrm{Ps} \rightarrow$ nothing mode [46], check of possible contribution from 2-photon mode [47-49] or from other exotic modes [50-52]. As a result it has been shown that no exotic modes can contribute to the anomaly, while contribution of ${ }^{\mathrm{T}} \mathrm{Ps} \rightarrow$ nothing mode is limited to $5.8 \times 10^{-4}$ of the regular decay.

The absence of theoretical explanation of $\lambda_{\mathrm{T}}$-anomaly encouraged Adkins et al. [53] to suggest experiments made in Japan [54] in 1995 as an alternative to the basic Michigan experiments. No doubt, high statistical accuracy of the Japanese measurements puts them on the same level with the basic experiments [35-38]. But all Michigan measurements possessed the property of a "full experiment", which in this particular case means no external influence could affect wave function of positronium. Such influence is inevitable due to electrodynamic nature of positronium and can be avoided only using special technique. In Japanese measurements [54] this was not taken into account and thus they do not possess property of "full experiment". Latest experiments of the Michigans [55], so-called Resolution of OrthopositroniumLifetime Pussle, as well do not possess property of "full experiment", because the qualitative another statement included external influence of electromagnetic field [56, 57].

As early as in 1993 Karshenboim [58] showed that QED had actually run out of any of its theoretical capabilities to explain orthopositronium anomaly.

Electric interactions and weak interactions were joined into a common electroweak interaction in the 1960's by com- monly Salam, Glashow, Weinberg, etc. Today's physicists attempt to join electroweak interaction and strong interaction (unfinished yet). They follow an intuitive idea that forces, connecting electrons and a nucleus, and forces, connecting nucleons inside a nucleus, are particular cases of a common interaction. That is the basis of our claim. If that is true, our claim is that orthopositronium atoms born in neon of different isotope contents $\left({ }^{22} \mathrm{Ne},{ }^{21} \mathrm{Ne},{ }^{20} \mathrm{Ne}\right)$ should be different from each other. There should be an effect of "inner" structure of neon nuclei if built by the Brightsen scheme, because the different proton-neutron contents built by different compositions of nucleon pairs. As soon as a free positron drags an electron from a neon atom, the potential of electro-weak interactions have changed in the atom. Accordingly, there in the nucleus itself should be re-distribution of strong interactions, than could be once as the re-building of the Brightsen pairs of nucleons there. So, lost electron of ${ }^{22} \mathrm{Ne}$ should have a different "inner" structure than that of ${ }^{21} \mathrm{Ne}$ or ${ }^{20} \mathrm{Ne}$. Then the life span of orthopositronium built on such electrons should be as well different.

Of course, we can only qualitatively predict that difference, because we have no exact picture of what really happens inside a "structurized" nucleus. Yet only principal predictions are possible there. However even in such case we vote for continuation of "isotope anomaly" experiments with orthopositronium in neon of different isotope contents. If further experiments will be positive, it could be considered as one more auxiliary proof that the Brightsen model is true.

\section{Acknowledgements}

We very much appreciate Dr. Robert Davic, the nephew of R. A. Brightsen, for comments about the Brightsen model.

\section{References}

1. Smarandache F. Matter, antimatter, and unmatter. Infinite Energy, 2005, v. 11, issue 62, 50-51.

2. Smarandache F. A new form of matter - unmatter, composed of particles and anti-particles. Progress in Physics, 2005, v. 1, 9-11.

3. Smarandache F. Verifying unmatter by experiments, more types of unmatter, and a quantum chromodynamics formula. Progress in Physics, 2005, v. 2, 113-116.

\section{UNMATTER BASIS EXPERIMENTS}

4. Gray L., Hagerty P., Kalogeropoulos T.E. Phys. Rev. Lett., 1971, v. 26, 1491-1494.

5. Carrol A.S., Chiang I.-H., Kucia T.F., Li K. K., Mazur P. O., Michael D. N., Mockett P., Rahm D. C., Rubinstein R. Phys. Rev. Lett., 1974, v. 32, 247-250.

6. Kalogeropoulos T. E., Vayaki A., Grammatikakis G., Tsilimigras T., Simopoulou E. Phys. Rev. Lett., 1974, v. 33, 1635-1637.

7. Chapiro I. S. Physics-Uspekhi, 1973, v. 109, 431. 
8. Bogdanova L.N., Dalkarov O.D., Chapiro I.S. Annals of Physics, 1974, v. 84, 261-284.

9. Cassidy D. B., Deng S. H. M., Greaves R. G., Maruo T., Nishiyama N., Snyder J. B., Tanaka H. K. M., Mills A.P. Jr. Phys. Rev. Lett., 2005, v. 95, No. 19, 195006.

10. Ball Ph. News Nature, 22 November 2005.

THE BRIGHTSEN MODEL

11. Brightsen R.A. Nucleon cluster structures in beta-stable nuclides. Infinite Energy, 1995, v. 1, no. 4, 55-56.

12. Brightsen R. A. Correspondence of the Nucleon Cluster Model with the Periodic Table of Elements. Infinite Energy, 1995/96, v. 1(5/6), 73-74.

13. Brightsen R. A. Correspondence of the Nucleon Cluster Model with the Classical Periodic Table of Elements. J. New Energy, 1996, v. 1(1), 75-78.

14. Brightsen R. A. The Nucleon Cluster Model and the Periodic Table of Beta-Stable Nuclides. 1996 (available online at http://www.brightsenmodel.phoenixrising-web.net).

15. Brightsen R. A. The nucleon cluster model and thermal neutron fission. Infinite Energy, 2000, v. 6(31), 55-63.

16. Brightsen R. A., Davis R. Appl. of the Nucleon Cluster Model to experimental results. Infinite Energy, 1995, v. 1(3), 13-15.

17. Bass R. W. Experimental evidence favoring Brightsen's nucleon cluster model. Infinite Energy, 1996, v. 2(11), 78-79.

SOME REVIEW PAPERS ON CLUSTER MODELS

18. Buck B., Merchant A.C., Perez S. M. Phys. Rev. C, 2005, v. 71(1), 014311-15.

19. Akimune H., Yamagata T., Nakayama S., Fujiwara M., Fushimi K., Hara K., Hara K. Y., Ichihara K., Kawase K., Matsui K., Nakanishi K., Shiokawa A., Tanaka M., Utsunomiya H., and Yosoi M. Physics of Atomic Nuclei, 2004, v. 67(9), 1721-1725.

20. Clustering Aspects of Nuclear Structure and Dynamics, Cluster'99, Proc. of the 7th Intern. Conf., 1999, Rab (Croatia).

21. Wuosmaa A. H., Betts R. R., Freer M., and Fulton B. R. Annual Review of Nuclear and Particle Science. 1995, v. 45, 89-131.

22. Bromley D. A. Clust. Aspects of Nucl. Structure, Proc. of the 4th Intern. Conf., Chester (UK), D. Reidel Publ., Dordrecht.

23. Horiuchi H. and Ikeda K. Cluster Models and Other Topics, Intern. Rev. of Nucl. Physics, 1986, v. 4, World Scientific, Singapore, 1-259.

CLUSTER MODELS THAT THE BRIGHTSEN MODEL BUILDS ON

24. Wheeler J. A. Phys. Rev., 1937, v. 52, 1083.

25. Wheeler J. A. Phys. Rev., 1937, v. 52, 1107.

26. Pauling L. Proc. Natl. Acad. Sci. USA, 1965, v. 54, no. 4, 989.

27. Pauling L. Science, 1965, v. 150, no. 3694, 297.

28. Pauling L. Revue Roumain de Physique, 1966, v. 11, no. 9/10, $825-833$.

ANOMALIES OF ORTHOPOSITRONIUM ANNIHILATION

29. Osmon P. E. Physical Review B, 1965, v. 138, 216.

30. Tao S. J., Bell J., and Green J.H. Proceedings of the Physical Society, 1964, v. 83, 453.
31. Levin B. M. and Shantarovich V. P. High Energy Chemistry, 1977, v. 11(4), 322-323.

32. Levin B. M. Soviet J. Nucl. Physics, 1981, v. 34(6), 917-918.

33. Levin B. M. and Shantarovich V.P. Soviet J. Nucl. Physics, 1984, v. 39(6), 855-856.

34. Levin B. M., Kochenda L. M., Markov A. A., and Shantarovich V. P. Soviet J. Nucl. Physics, 1987, v. 45(6), 1119-1120.

35. Gidley D. W., Rich A., Sweetman E., and West D. Physical Review Letters, 1982, v. 49, 525-528.

36. Westbrook C.I., Gidley D.W., Conti R.S., and Rich A. Physical Review Letters, 1987, v. 58, 1328-1331.

37. Westbrook C.I., Gidley D.W., Conti R.S., and Rich A. Physical Review A, 1989, v. 40, 5489-5499.

38. Nico J. S., Gidley D. W., Rich A., and Zitzewitz P. W. Physical Review Letters, 1990, v. 65, 1344-1347.

39. Caswell W. E. and Lepage G. P. Phys. Rev. A, 1979, v. 20, 36.

40. Adkins G. S. Ann. Phys. (N.Y.), 1983, v. 146, 78.

41. Adkins G. S., Salahuddin A. A., and Schalm K.E. Physical Review A, 1992, v. 45, 3333-3335.

42. Adkins G.S., Salahuddin A. A., and Schalm K.E. Physical Review A, 1992, v. 45, 7774-7780.

43. Levin B. M. Physics of Atomic Nuclei, 1995, v. 58(2), 332-334.

44. Holdom B. Physics Letters B, 1986, v. 166, 196-198.

45. Glashow S. L. Physics Letters B, 1986, v. 167, 35-36.

46. Atoyan G. S., Gninenko S. N., Razin V. I., and Ryabov Yu. V. Physics Letters B, 1989, v. 220, 317-320.

47. Asai S., Orito S., Sanuki T., Yasuda M., and Yokoi T. Physical Review Letters, 1991, v. 66, 1298-1301.

48. Gidley D.W., Nico J.S., and Skalsey M. Physical Review Letters, 1991, v. 66, 1302-1305.

49. Al-Ramadhan A. H. and Gidley D. W. Physical Review Letters, 1994, v. 72, 1632-1635.

50. Orito S., Yoshimura K., Haga T., Minowa M., and Tsuchiaki M. Physical Review Letters, 1989, v. 63, 597-600.

51. Mitsui T., Fujimoto R., Ishisaki Y., Ueda Y., Yamazaki Y., Asai S., and Orito S. Phys. Rev. Lett., 1993, v. 70, 2265-2268.

52. Skalsey M. and Conti R.S. Phys. Rev. A, 1997, v. 55(2), 984.

53. Adkins G.S., Melnikov K., and Yelkhovsky A. Phys. Rev. A, 1999, v. 60(4), 3306-3307.

54. Asai S., Orito S., and Shinohara N. Physics Letters B, 1995, v. $357,475-480$.

55. Vallery R. S., Zitzewitz P. W., and Gidley D. W. Phys. Rev. Lett., 2003, v. 90, 203402.

56. Levin B. M. arXiv: quant-ph/0303166.

57. Levin B. M. CERN EXT-2004-016.

58. Karshenboim S. G. Yadern. Fizika, 1993, v. 56(12), 155-171. 